\title{
Escala de Procrastinação Ativa: evidências de validade fatorial e consistência interna
}

\author{
Valdiney V. Gouveia - Universidade Federal da Paraíba, João Pessoa, Brasil \\ Viviany da Silva Pessoa - Universidade Federal da Paraíba, João Pessoa, Brasil \\ Marcio de Lima Coutinho - Universidade Federal da Paraíba, João Pessoa, Brasil \\ Iany C. da S. Barros - Centro Universitário de João Pessoa, João Pessoa, Brasil \\ Aline Arruda da Fonseca - Faculdade Maurício de Nassau, João Pessoa, Brasil
}

\begin{abstract}
Resumo
Este estudo objetivou adaptar para o contexto brasileiro a Escala de Procrastinação Ativa (EPA), reunindo evidências de validade e precisão. Participaram 216 universitários, com idade média de 25,9 anos $(D P=8,40)$. Estes responderam a EPA e perguntas demográficas. Os resultados de análises fatoriais confirmatórias, comparando diferentes modelos, permitiram comprovar como mais adequada a estrutura com quatro fatores: preferência por pressão $(\alpha=0,72)$, babilidade em cumprir praz̧os $(\alpha=0,72)$, satisfação com os resultados $(\alpha=0,66)$ e decisão intencional $(\alpha=0,61)$, que apresentou indicadores de ajuste aceitáveis $(G F I=0,91$ e $R M S E A=0,06)$. Tais fatores apresentaram evidências de validade convergente, porém dois deles não se diferenciaram satisfatoriamente. Concluiu-se que estes achados apoiam a adequação psicométrica deste instrumento, que apresenta evidências de validade e precisão.

Palavras-chave: Procrastinação; Validade; Precisão; Escala.
\end{abstract}

Escala de Procrastinación Activa: evidencias de validez factorial y confiabilidad

\begin{abstract}
This article aimed to adapt for the Brazilian context the Scale of Active Procrastination (SAP), gathering evidence of validity and reliability. Participants were 216 undergraduate students, with a mean age of 25.9 years $(S D=8.40)$. They answered to the $S A P$ and demographic questions. Results of confirmatory factor analyses, after testing alternative models, allowed to prove as more appropriate the structure with four factors: preference for pressure $(\alpha=0.72)$, ability to meet deadlines $(\alpha=0.72)$, satisfaction with the results $(\alpha=0.66)$, and intentional decision $(\alpha=0.61)$, which presented acceptable fit indexes (GFI=0.91 and RMSE $A=0.06)$. Such factors showed evidence of convergent validity, but two of them did not differ sufficiently. In conclusion, these findings support the psychometric adequacy of this instrument, which shows evidence of validity and reliability.

Keywords: Procrastination; Validity; Reliability; Scale.
\end{abstract}

Active procrastination scale: evidence of factorial validity and reliability

\begin{abstract}
Resumen
Este estudio tuvo como objetivo adaptar la Escala de Procrastinación Activa (EPA) para el contexto brasileño, reuniendo evidencias de validez y confiabilidad. Participaron 216 estudiantes universitarios, con promedio de edad de 25,9 años $(D T=8,40)$, que contestaron la EPA y preguntas demográficas. Los resultados de análisis factoriales confirmatorios, comparando diferentes modelos, permitieron comprobar que la estructura con cuatro factores fue la más adecuada: preferencia por presión $(\alpha=0,72)$, capacidad para cumplir plazos $(\alpha=0,72)$, satisfacción con los resultados $(\alpha=0,66)$ y decisión intencional $(\alpha=0,61)$, que presentaron indicadores de ajuste aceptables $(G F I=0,91$ y $R M S E A=0,06)$. Estos factores presentaron evidencias de validez convergente, aunque dos de ellos no se diferenciaron satisfactoriamente. Se concluyó que estos hallazgos soportan la adecuación psicométrica de este instrumento, que muestra evidencias de validez y confiabilidad.

Palabras-clave: Procrastinación; Validez; Confiabilidad; Escala.
\end{abstract}

\section{Introdução}

Deus, dai-me continência e castidade, mas não agora Santo Agostinho

"Não deixe para amanhã o que você pode fazer hoje". Certamente, esta frase já foi dita muitas vezes. A simples lembrança desse ditado popular sugere a evitação do ato de procrastinar, isto é, adiar, delongar, demorar e transferir para outro dia o que poderia ser prontamente realizado. Se, por um lado, as pessoas são educadas a ver a procrastinação de modo negativo, por outro, estima-se que o ato de adiar atividades seja mais frequente do que se imagina, uma tendência que acompanha o homem há séculos, como se depreende das palavras de Santo Agostinho. Estudos indicam que a procrastinação é um fenômeno motivacional, apresentando elementos individuais e contextuais (Steel, 2007). De acordo com Potts (1987), esse fenômeno ocorre em ao menos $95 \%$ da população em geral e, cronicamente entre $15 \%$ a $20 \%$ de adultos e $33 \%$ a $50 \%$ de estudantes.

Até o início dos anos 2000, a literatura vinha apresentando estudos sobre a procrastinação unicamente como um gerador de desconforto emocional, 
um aspecto patológico do comportamento que se presumia interferir no desempenho individual ou grupal, estando presente cotidianamente em diversos contextos (e.g., vida escolar / acadêmica, trabalho, relações interpessoais, cuidados com a saúde). Os elementos caracterizadores da procrastinação foram identificados, por exemplo, como aversão à tarefa, atraso da tarefa, baixa autoeficácia e impulsividade, assim como distração, autocontrole, falta de informação e organização (Akerlof, 1991; Boice, 1996; Ellis \& Knaus, 1977; Ferrari, Johnson \& McCown, 1995; Hamasaki \& Kerbauy, 2001; Knaus, 2000; Milgram, Sroloff \& Rosenbaum, 1988; Strongman \& Burt, 2000).

Tendo como base esse panorama, Silva e Yu (2009) destacaram dois pontos interessantes. Primeiro, que a procrastinação poderia causar a depressão, a ansiedade extrema, o estresse, o déficit de atenção e a desorganização mental. Segundo, que o fator tempo, que era então visto como central no conceito de procrastinação, não estaria implicado na evitação da tarefa, mas sim em como o indivíduo gerenciava o tempo para concluir com sucesso uma atividade. EsSe segundo ponto sinaliza que, embora predominantemente tenham sido destacados aspectos negativos da procrastinação, podem ser reconhecidos igualmente aspectos positivos que caracterizam essa ação (Choi \& Moran, 2009; Chu \& Choi, 2005). Faz-se necessário observar que o papel da procrastinação nesta configuração ou faceta mais positiva vem despertando interesse de profissionais e pesquisadores de diversas áreas da psicologia, como social, educacional e organizacional, mas também daqueles de outros campos de atuação, como marketing, economia e engenharia, por exemplo (Bernold, 2007).

Os primeiros achados a respeito indicaram que nem todos os comportamentos de procrastinação são inadequados, prejudiciais ou levam a consequências indesejáveis. Nesse sentido, são diferenciados dois tipos de procrastinadores: os passivos e os ativos. Os passivos são considerados procrastinadores tradicionais, paralisando suas ações em virtude de indecisões de agir sobre a realidade, deixando de executar tarefa em tempo hábil. Em contrapartida, os ativos apresentam características desejáveis de comportamentos e atitudes, preferindo trabalhar sob pressão, tomando decisões, controlando o tempo e a autoeficácia para melhor realização de tarefas.

Neste contexto, a procrastinação não se apresenta apenas como um componente de administração de tempo, pois ela envolve fatores que também estão relacionados com fenômenos comportamentais, afetivos e cognitivos, assim como destaca a noção de que uma tarefa pode ser adiada até sua data-limite e mesmo assim ser bem executada, obtendo resultados positivos, despertando no agente sensações agradáveis de capacidade de realização e autoeficácia (Bui, 2007; Fee \& Tangney, 2000). Nessa direção, a partir de estudo com estudantes universitários no Canadá, oriundos de diferentes culturas, Chu e Choi (2005) indicaram quatro variáveis que fundamentam a procrastinação: estresse, depressão, satisfação com a vida e nível de desempenho. Como resultado desse estudo, mapearam uma estrutura multidimensional, reunindo quatro fatores que definem a procrastinação ativa: preferência por pressão (PPP), decisão intencional (DI), habilidade em cumprir prazos (HCP) e satisfação com os resultados (SCR), fundamentando uma nova medida desse construto.

\section{Escala de Procrastinação Ativa (EPA)}

Ao construir uma medida para avaliar a procrastinação ativa, como definida por Chu e Choi (2005), Choi e Moran (2009) testaram uma estrutura que agrupou as quatro dimensões previamente descritas, correspondendo à Escala de Procrastinação Ativa $(E P A)$, composta por 16 itens igualmente distribuídos nesses fatores, tendo sido observados indicadores de ajuste adequados ( $C F I=0,92$ e $R M S E A=0,058)$. Esses autores também testaram modelos alternativos, compreendendo três e duas dimensões, porém tais modelos não apresentaram indicadores de ajuste superiores aos previamente relatados, estando abaixo do comumente aceito. Portanto, decidiram assumir a estrutura tetrafatorial, cujos fatores são mais bem descritos a seguir:

Preferência por pressão (PPP). Esta característica considera o estresse como elemento preponderante, que é motivado pela necessidade intrínseca de lidar com o desafio e por demandas externas para completar a tarefa no tempo determinado. Segundo Freedman e Edwards (1988), essa pressão pode desenvolver sentimentos de desafio, que não são necessariamente negativos no plano psicológico. Os seguintes itens permitem representá-lo: "É realmente difícil para mim trabalhar sabendo que os prazos estão próximos" e "Fico chateado e relutante para agir quando sou forçado a trabalhar sob pressão".

Satisfação com os resultados (SCR). Os indivíduos que procrastinam tendem a se motivar quando, sob pressão, executam as tarefas em tempo hábil, obtendo resultados satisfatórios. Portanto, procrastinadores ativos decidem intencionalmente adiar suas tarefas, postergando para o último momento sua execução com o intuito de utilizar 
eficazmente o seu tempo para obter um resultado gratificante. Dois dos itens que representam este fator são: "Meu desempenho tende a piorar quando tenho que correr para cumprir prazos" e "Não me saio bem se tenho que fazer minhas atividades às pressas".

Habilidade em cumprir prazos (HCP). Os procrastinadores ativos são capazes de estipular corretamente um tempo mínimo necessário para concluir uma tarefa, utilizando estratégias de enfrentamento sob estresse para atingir o sucesso. Podem descrever adequadamente este fator os seguintes itens: "Quando começo alguma atividade, tenho dificuldade em terminá-la" e "Com frequência não consigo cumprir metas que estabeleço para mim mesmo".

Decisão intencional (DI). Os procrastinadores tendem a alternar de uma atividade para outra sem muito planejamento ou organização do tempo. Estão dispostos a adiar tarefas que planejaram fazer, mudando a sua programação mesmo em curto prazo. Assim, em vez de serem fixadas em sua rotina atividades pré-agendadas, eles intencionalmente remodelam suas atividades de forma a responder às mudanças externas. Dois itens que permitem representar este fator são: "Adio de propósito minhas atividades para usar meu tempo mais eficientemente" e "Adio intencionalmente o meu trabalho para aumentar minha motivação".

Por fim, há que esclarecer que os itens dos fatores PPP, SCR e HCP foram redigidos no sentido negativo com o intuito de favorecer a compreensão do respondente. Por outro lado, aqueles do fator DI foram escritos de forma positiva. Portanto, demanda-se inverter as pontuações daqueles fatores, mas não deste.

\section{Medindo a procrastinação ativa no contexto brasileiro \\ Considerando o contexto brasileiro, Kerbauy} (1997) procurou enquadrar os estudos sobre a procrastinação em três categorias principais: (1) hábitos de estudo, que se referem aos atos de não concluir trabalhos no prazo determinado ou não estudar para provas; (2) contexto clínico, que estuda como a procrastinação interfere na tomada de decisões para resoluções importantes na vida, como trabalho, casamento, amor e família; e (3) vida cotidiana, que abrange questões gerais do dia a dia, como pontualidade e definição de prioridades. Posteriormente, Enumo e Kerbauy (1999) apresentaram vários estudos utilizando escalas que permitem avaliar a procrastinação, como a procrastinação acadêmica, sendo utilizada a Procrastination Assessement Scale - Students (PASS; Solomon \& Rothblum, 1984).
Conforme se observa em Enumo e Kerbauy (1999), os estudos sobre procrastinação, no cenário brasileiro, refletem uma concepção passiva, evidenciando característica consideradas frequentes entre os brasileiros, como adiar tarefas e decisões. No estudo feito por essas autoras, visando abordar o conceito de procrastinação, o comportamento de adiar e suas consequências, participaram 50 transeuntes e 22 estudantes de psicologia de uma universidade pública da cidade de Vitória (ES). Seus resultados apoiaram a concepção de que as pessoas costumam procrastinar, sendo o conceito correspondente baseado na ideia de deixar para depois ou atrasar o início de tarefas diversas (e.g., afazeres domésticos, estudar, ir ao médico e dentista). Outros resultados interessantes desse estudo foram quanto ao gênero. As mulheres relataram menos procrastinação com os compromissos sociais e financeiros. Os homens, por sua vez, relataram menos procrastinação com os cuidados pessoais, como fazer ginástica e barbar-se diariamente. A generalização desses resultados é, no entanto, limitada em razão do número reduzido de participantes e a influência presumível da desejabilidade social em suas respostas.

Com o intuito de encontrar estudos brasileiros acerca da procrastinação ativa, realizaram-se buscas em bases de dados (BVS, 2013; PsychINFO, 2013) utilizando como palavras-chave "procrastinação" e "medida", não sendo encontrada qualquer referência sobre procrastinação ativa. Estima-se que este cenário reflete uma escassez de estudos a respeito na literatura brasileira, apesar de reconhecer que podem existir dissertações e teses que vêm sendo dedicadas a essa temática.

Em resumo, parece evidente que a procrastinação não apenas encerra uma conotação negativa, mas pode igualmente favorecer elementos positivos, como no caso de sua dimensão ativa. Esse é um aspecto psicológico importante para compreender o comportamento de procrastinar, que pode resultar em ações exitosas por parte de alguns indivíduos. Entretanto, ainda são escassos os instrumentos desenvolvidos para a avaliação da procrastinação ativa, não tendo sido identificada qualquer medida a respeito na realidade brasileira. Desse modo, o objetivo do presente estudo foi adaptar para este contexto a escala desenvolvida por Choi e Moran (2009), buscando conhecer evidências de sua validade (validades fatorial, convergente e discriminante) e precisão (homogeneidade e consistência interna). 


\section{Método}

\section{Participantes}

Participaram 216 estudantes universitários de duas instituições públicas de ensino superior de João Pessoa (PB). Estes tinham idades variando de 18 a 70 anos $(M=25,9 ; D P=8,40)$, sendo a maioria do sexo feminino $(75 \%)$, solteira $(69 \%)$ e católica $(53,2 \%)$. Tratou-se de uma amostra não probabilística, constituída por pessoas que, convidadas, concordaram em colaborar voluntariamente com a pesquisa.

\section{Instrumento}

Os participantes responderam a um questionário composto por medidas para avaliar a procrastinação ativa, atitudes frente à fofoca (Gouveia, Sousa, Albuquerque-Souza, Sá-Serafim \& Gonçalves, 2011), desejabilidade social (Gouveia, Guerra, Sousa, Santos \& Costa, 2009) e valores humanos (Gouveia, 2013). Incluíram-se, ademais, nove perguntas gerais, sendo seis demográficas (idade, estado civil, horas de sono por dia, religião, nível de religiosidade e sexo) e três acadêmicas (CRE, horas de estudo por semana e percepção de bom estudante). Considerando os propósitos deste estudo, descreve-se unicamente a medida de procrastinação, como segue:

Escala de Procrastinação Ativa (EPA; Choi \& Moran, 2009). Originalmente elaborada em inglês, esta medida é composta por 16 itens igualmente distribuídos em quatro fatores que cobrem aspectos cognitivos (e.g., Tenho dificuldade de concluir o que eu iniciei), afetivos (e.g., Fico chateado e relutante quando sou forçado a trabalhar sob pressão) e comportamentais (e.g., Meu desempenho tende a piorar quando tenho que correr para cumprir prazos). Estes são respondidos em escala tipo likert, de sete pontos, variando de 1 (Totalmente não verdadeiro) a 7 (Totalmente verdadeiro).

A versão original desta escala foi traduzida para o português por duas psicólogas bilíngues, submetida à apreciação de um terceiro psicólogo também bilíngue. Alcançando acordo quanto à melhor tradução, procedeu-se à sua validação semântica, considerando-se a participação de dez estudantes universitários do primeiro período de psicologia de uma IES pública, procurando avaliar a adequação dos itens, da escala de resposta e das instruções sobre como responder. Não tendo sido verificada qualquer alteração, definiu-se a versão experimental em português, que poderá ser obtida sob solicitação a um dos autores deste estudo (http:/vvgouveia.net).

\section{Procedimento}

Dois colaboradores devidamente capacitados ficaram responsáveis pela coleta de dados. Após a autorização do professor da disciplina, os aplicadores informaram que se tratava de uma pesquisa sobre comportamentos sociais. Uma vez tendo concordado em colaborar, os participantes assinaram um Termo de Consentimento Livre e Esclarecido, baseado nos preceitos éticos vigentes para a realização de pesquisas com seres humanos, defendidos pela Resolução 466/12, que assegurava o caráter voluntário, anônimo e confidencial da participação, permitindo a desistência do estudo a qualquer momento, sem penalização. A aplicação dos questionários se deu em contexto coletivo de sala de aula, porém com cada participante respondendo individualmente. Os aplicadores permaneceram em sala para esclarecer eventuais dúvidas quanto à forma de responder, não tecendo qualquer comentário sobre o conteúdo dos itens. Finalmente, os pesquisadores agradeceram a participação de todos e informaram um endereço eletrônico onde os interessados poderiam ter acesso aos resultados gerais da pesquisa. As pessoas levaram em média 20 minutos para concluir suas participações.

\section{Análise dos dados}

Os dados foram analizados com o $P A S W$ (versão 18). Além de estatísticas descritivas, foram calculadas correlações $r$ de Pearson e índices de consistência interna (alfa de Cronbach) dos fatores resultantes. Para comprovação da estrutura fatorial da EPA, realizaram-se análises fatoriais confirmatórias $(C F A s)$ com o AMOS (versão 7). Esse tipo de análise é adequado para comprovar se determinada estrutura fatorial se adequa aos dados, pois permite: (a) a especificação e comparação de modelos teoricamente relevantes e (b) a identificação de fatores de primeira e segunda ordens. Os seguintes indicadores de ajuste foram considerados (Byrne, 2010; Tabachnick \& Fidell, 2013):

- O Qui-quadrado $\left(\chi^{2}\right)$. Comprova a probabilidade de o modelo se ajustar aos dados. Um valor de $\chi^{2}$ significativo indica discrepância entre os dados e o modelo testado. Esse indicador é influenciado pelo tamanho da amostra. A propósito, costuma-se ter em conta a razão $\chi^{2}$ /g.l. (qui-quadrado / graus de liberdade), considerada uma qualidade de ajuste subjetiva (Byrne, 2010). Um valor inferior a 5,00 pode ser interpretado como indicando a adequação do modelo teórico para descrever os dados, aceitando-se como mais favoráveis aqueles entre 2 e 3 . 
- O Índice de Qualidade do Ajuste (Goodness-of-Fit Index, GFI) e o Índice de Qualidade do Ajuste Ponderado (Adjusted Goodness-of-Fit Index, AGFI), cujos valores variam de 0 a 1 , com aqueles próximos a 0,90 indicando ajustamento satisfatório.

- Índice de Ajuste Comparativo (Comparative Fit Index, CFI) é um índice adicional de ajuste do modelo aos dados, com valores mais próximos de 1 indicando melhor ajuste; 0,90 pode ser considerado como referência para aceitar o modelo.

- Raíz quadrada média do erro de aproximação (Root Mean Square Error of Approximation, RMSEA). Recomendam-se valores próximos a 0,05 , preferentemente abaixo de 0,08, embora se aceitem aqueles de até 0,10, desde que não ultrapassem o intervalo de confiança respectivo de 90\% (IC $90 \%)$.

- Índice de trasvalidação esperado (ECVI) e Critério de informação de Akaike (CAIC) têm a finalidade de comparar os modelos alternativos; valores de ECVI e CAIC mais baixos indicam melhor ajuste do modelo analisado. Além desses índices, costuma-se considerar a diferença de $\chi^{2}$ e seus respectivos graus de liberdade $\left[\Delta \chi^{2}\right.$ (g.l.) $]$ para decidir acerca do mais ajustado; um valor $\Delta \chi^{2}$ com um $p<0,05$ ratifica melhor ajuste do modelo com menor qui-quadrado.

Por fim, procurou-se conhecer evidências de validade de construto (validade convergente, validade discriminante e confiabilidade composta). Neste caso, calculou-se a variância média extraída (VME), que indica o quanto o construto explica o conjunto de itens, sendo um valor 0,50 indicativo de validade convergente. Se tomada em conta a raiz quadrada da VME de cada fator, comparadando-a com a correlação entre os pares de fatores $(\Phi)$, se seu valor for superior, indica-se validade discriminante. Por fim, a confiabilidade composta (CC) é um indicador complementar de precisão da medida, comparável ao alfa de Cronbach, porém sem fazer as mesmas pressuposições de independência de erros de medida e tau equivalência dos itens. No caso, demanda-se que a CC seja igual ou superior a 0,60 para atestar este parâmetro (Škerlavaj \& Dimovski, 2009).

\section{Resultados}

\section{Estrutura Fatorial da EPA: Testando Modelos Alternativos}

Com a finalidade de testar modelos alternativos à estrutura multidimensional apresentada por Choi e Moran (2009), e tendo assegurado o procedimento de limpeza do banco de dados e eliminação de casos perdidos, optou-se pela modelagem por equações estruturais, considerando a matriz de variâncias-covariâncias e adotando o estimador ML (Maximum Likelihood). O modelo original, tetrafatorial, em que os itens foram igualmente distribuídos nos fatores (HCP, PPP, DI e SCR), foi inicialmente testado $\left(M_{1}\right)$. Posteriormente, testou-se um modelo bifatorial, que agrupou os itens segundo a similaridade de seus fatores, isto é, HCP + DI e SCR+PPP, tendo em conta a matriz de correlação dos estudos de elaboração dessa medida $\left(M_{2}\right)$; e, finalmente, comprovou-se um modelo unifatorial $\left(M_{3}\right)$, em que todos os itens foram agrupados em um fator geral de procrastinação (DI + HCP + PPP + SCR). Os resultados destas análises são apresentados na Tabela 1.

Como é possível observar nos indicadores de ajuste, o último modelo $\left(M_{3}\right)$, que supõe unifatorialidade, deve ser descartado, considerando que seus indicadores de ajuste foram abaixo dos recomendados.

Tabela 1. Indicadores de Ajuste dos Modelos Alternativos

\begin{tabular}{lcccccccc}
\hline Modelos & $\chi^{2}(\mathrm{gl})$ & GFI & AGFI & CFI & $\begin{array}{c}\text { RMSEA } \\
(\mathrm{IC} 90 \%)\end{array}$ & $\begin{array}{c}\text { ECVI } \\
(\mathrm{IC} 90 \%)\end{array}$ & CAIC & $\Delta \chi^{2}$ \\
\hline $\mathrm{M}_{1}$. Tetrafatorial & $182,43(98)$ & 0,91 & 0,87 & 0,90 & $\begin{array}{c}0,063 \\
(0,049-0,077)\end{array}$ & $\begin{array}{c}1,20 \\
(1,04-1,40)\end{array}$ & 424,69 & ---- \\
$\mathrm{M}_{2}$. Bifatorial & $492,23(103)$ & 0,71 & 0,62 & 0,53 & $\begin{array}{c}0,133 \\
(0,121-0,144)\end{array}$ & $\begin{array}{c}2,60 \\
(2,29-2,94)\end{array}$ & 702,61 & $309,80(5)^{*}$ \\
\hline $\mathrm{M}_{3}$. Unifatorial & $492,70(104)$ & 0,71 & 0,63 & 0,53 & $\begin{array}{c}0,132 \\
(0,120-0,144)\end{array}$ & $\begin{array}{c}2,59 \\
(2,29-2,93)\end{array}$ & 696,71 & $310,27(6)^{*}$ \\
\hline
\end{tabular}

Nota: $* p<0,001$ 
O segundo modelo $\left(M_{2}\right)$, admitindo uma estrutura bifatorial, apresentou indicadores de ajuste melhores que o anterior, podendo ser admitido como meritório, porém não estritamente apropriado. $\mathrm{O}$ primeiro modelo $\left(M_{1}\right)$, reproduzindo a estrutura original da medida de procrastinação, mostrou-se mais adequado, reunindo indicadores de ajuste que podem ser aceitos. Portanto, esta estrutura, admitindo quatro fatores para representar a procrastinação positiva (ativa), que foi a teoricamente esperada, apresentou os seguintes indicadores de ajuste: $\chi^{2}(98)=182,43, \chi^{2} / \mathrm{gl}=1,86, G F I=0,91$, $A G F I=0,87, C F I=0,90$ e $R M S E A=0,063]$. Quando os qui-quadrados dos três modelos foram comparados, evidenciou-se a adequação do tetrafatorial em relação ao bifatorial $\left[\Delta \chi^{2}(5)=309,80, p<0,001\right]$ e unifatorial $\left[\Delta \chi^{2}(6)=310,27, p<0,001\right]$. Os respectivos CAICs $(424,69,702,61$ e 696,71$)$ e ECVIs $(1,20,2,60$ e 2,59) corroboram a superioridade do $M_{\text {. }}$.

Considerando os achados previamente descritos, parece evidente que o modelo original, isto é, tetrafatorial, reuniu os melhores indicadores de ajuste. Nesse sentido, representa-se na Figura 1 a estrutura deste modelo, indicando as saturações (pesos de regressão padronizados) de cada item em seu respectivo fator. Ressalta-se que todas as saturações foram estatisticamente diferentes de zero $\left(\lambda \neq 0 ; z^{>} 3, p<0,001\right)$, variando de 0,34 (Item 15. Estou frequentemente atrasado quando vou fazer algo) a 0,77 (Item 5. É realmente difícil para mim trabalhar sabendo que os prazos estão próximos).

\section{Homogeneidade e consistência interna}

A homogeneidade dos itens foi avaliada por meio da correlação item-total corrigida para cada fator. Este parâmetro variou de 0,22 (item 15) a 0,49 (item 13) para o fator DI, 0,49 (item 11) a 0,60 (item 6) para o HCP, 0,37 (item 1) a 0,55 (item 5) para o SCR e, finalmente, 0,48 (item 04) a 0,58 (item 14) para o fator PPP. No caso da consistência interna, esta foi verificada por meio do alfa de Cronbach $(\alpha)$, tendo os fatores apresentado os seguintes coeficientes: 0,61(DI), 0,73 (HCP), 0,66 (SCR) e 0,72 (PPP). Considerando o conjunto total de itens, a escala apresentou consistência interna $(\alpha)$ de 0,78 . Os resultados são mostrados na Tabela 2.

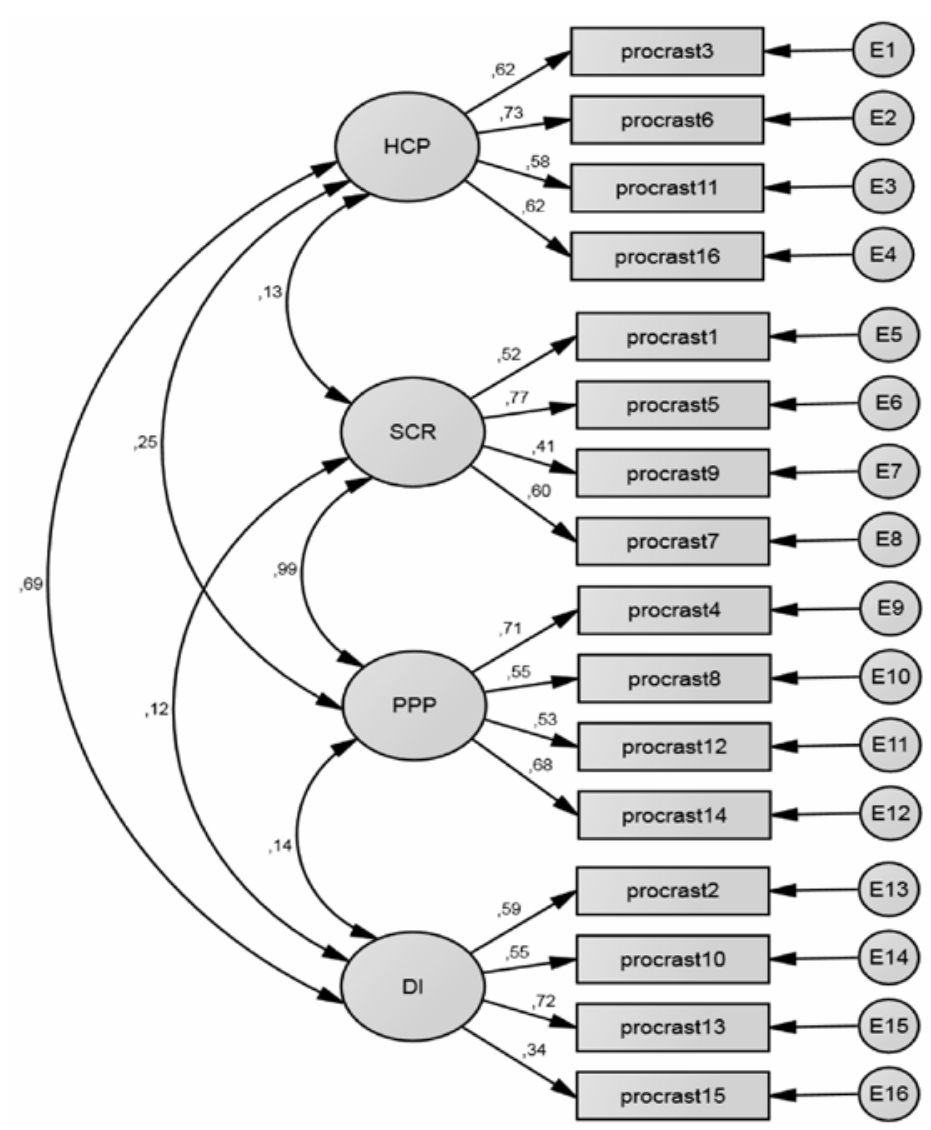

Figura 1. Modelo explicativo da análise confirmatória 


\section{Evidências de validade convergente-discriminante e confiabilidade composta}

Nesta oportunidade, procurou-se reunir evidências complementares de validade de construto (validade convergente, validade discriminante e confiabilidade composta). Desse modo, utilizaram-se os pesos de regressão (valores padronizados) para computar os indicadores correspondentes. No caso da validade convergente, observaram-se os seguintes coeficientes: $S C R$ $(0,60), P P P(0,63), D I(0,57)$ e HCP $(0,65)$; para checar evidências de validade discriminante, comparou-se a raiz quadrada de cada um desses coeficientes com a correlação entre cada par de fatores. Como é possível observar na Tabela 2, unicamente não se verificou este parâmetro entre os fatores SCR $(\sqrt{ } \mathrm{VME}=0,77)$ e PPP $(\sqrt{ } \mathrm{VME}=0,79) \quad(\Phi=0,99)$. Por fim, constatou-se que todos os fatores reuniram evidências de confiabilidade composta, tendo os coeficientes variado de 0,64 (DI) a 0,74 (HCP).

\section{Discussão}

O objetivo principal deste artigo foi adaptar para o contexto brasileiro a Escala de Procrastinação Ativa, conhecendo evidências de validade e precisão. Confiase que este tenha sido alcançado. No entanto, faz-se necessário ressaltar limitações potenciais deste estudo, a exemplo do tamanho e da natureza da amostra, sendo de um número reduzido e não probabilística, com a participação unicamente de estudantes do ensino superior de uma cidade do Nordeste brasileiro. Desse modo, os achados não podem ser generalizados quanto à tendência para a procrastinação dos participantes deste país. Não obstante, esclarece-se, o número de participantes foi suficiente para realizar as análises estatísticas (Clark \& Watson, 1995), permitindo concluir acerca das evidências psicométricas da medida em pauta. Nesse sentido, procura-se descrever os principais achados, tratando-os em termos dos parâmetros observados.

\section{Evidências de validade de construto}

Com base na comparação dos três modelos propostos, isto é, (a) unifatorial: considerando todos os itens saturando em um único fator, (b) bifatorial: partindo da associação empírica entre os fatores, de acordo com estudo prévio (Choi \& Moran, 2009), unindo HCP e DI, por um lado, e SCR e PPP, por outro, e, finalmente, (c) o tetrafatorial, que presume a saturação dos itens em cada um dos quatro fatores propostos (HCP, PPP, DI e SCR), procedeu-se à comparação. Claramente, este último modelo, coerente com os achados de Choi e Moran (2009), revelou-se o mais adequado, reunindo indicadores de ajuste que podem ser considerados satisfatórios, como GFI maior que 0,90 e RMSE $A$ menor que 0,08 (Byrne, 2010).

Esta estrutura reuniu quatro fatores, cada um representado por quatro itens, todos apresentando saturações que se aproximam de $|0,50|$, ponto de corte referido na literatura (Hair, Black, Babin, Anderson \& Tatham, 2009), sendo diferentes de zero, revelando sua adequação como representação do traço latente correspondente (Byrne, 2010; Clark \& Watson, 1995). Contudo, ao observar as caractrísticas do item com a menor saturação (Item 15. Por escolha própria, concluo a maioria das minhas atividades faltando pouco tempo para acabar o prazo), constatou-se que este apresentou problemas; concretamente, apesar de pertencente ao fator DI, correlacionou-se mais fortemente com os itens do fator HCP. Quiçá uma falha na construção semântica deste item compreenda a justificativa mais plausível, pois enfoca mais a habilidade para cumprir prazo do que a submissão a contexto de pressão.

Tabela 2. Confiabilidade composta, variância média extraída e correlação interfatores

\begin{tabular}{lccccccc}
\hline Fatores & $\alpha$ & CC & VME & VVME & \multicolumn{2}{c}{ Correlações $\Phi(\mathrm{r})$} \\
\hline 1. SCR & 0,66 & 0,67 & 0,60 & 0,77 & & & \\
2. PPP & 0,72 & 0,72 & 0,63 & 0,79 & $0,99\left(0,68^{* *}\right)$ & & $0,14(0,06)$ \\
3. DI & 0,61 & 0,64 & 0,57 & 0,75 & $0,12(0,07)$ & 0,14 & \\
4. HCP & 0,73 & 0,74 & 0,65 & 0,81 & $0,13(0,12)$ & $0,25\left(0,18^{* *}\right)$ & $0,69\left(0,50^{* *}\right)$ \\
\hline
\end{tabular}

Notas: SCR (satisfação com os resultados), PPP (preferência porpressão), DI (Decisão Intencional), HCP (habilidade em cumprir prazos); $a=$ alfa de Cronbach; $C C=$ Confiabilidade Composta; $V M E=$ Variância Média Extraída; $\sqrt{ } V M E=$ Raiz quadrada da Variância Média Extraída; $\Phi=$ correlação entre os construtos latentes, resultado da análise fatorial confirmatória; $r=$ correlação de Pearson. 
Portanto, caberá rever seu sentido ou mesmo elaborar outro item.

Com o fim de complementar as evidências de validade de construto, procurou-se focar na medida em si, tratando de seu conjunto de itens, avaliando o quanto poderiam representar adequadamente os fatores (construtos) respectivos, correspondendo à validade convergente, e se poderiam ser diferenciados entre si, evidenciando mais algo próprio, inerente a cada fator, do que compartilhado com os demais (validade discriminante). Coerente com o esperado, os quatro fatores reuniram evidências do primeiro tipo de validade, uma vez que suas $V M E s$ foram superiores a 0,50 ; entretanto, dois deles não mostraram evidências do segundo tipo de validade (SCR e PPP), pois a correlação entre ambos foi superior às respectivas raízes quadradas de suas VMEs (Hair e cols., 2009; Škerlavaj \& Dimovski, 2009). Este achado carece de replicação, uma vez que a estrutura originalmente proposta se mostrou a mais adequada, admitindo a legitimidade dos quatro fatores.

\section{Evidências de precisão}

Inicialmente, destaca-se que a versão original desta medida apresentou alfas de Cronbach $(\alpha)$ que alcançaram o ponto de corte comumente desejado na literatura (0,70; Nunnally, 1991; Pasquali, 2003). Porém, isso não se constatou no presente estudo; dois fatores atenderam a esta recomendação (HCP e PPP), porém outros dois apresentaram consistência interna $(\alpha)$ algo inferior, embora acima de 0,60 , justificando empregar esta medida em contexto de pesquisa (Clark \& Watson, 1995). Vale ressaltar, entretanto, que o número de itens por fator, que é de quatro, pode ter contribuído negativamente para este parâmetro (Pasquali, 2003).

$\mathrm{Na}$ direção do anteriormente comentado, quando se considera o parâmetro de cada item, tratando em termos de sua correlação com a pontuação total da medida (homogeneidade), encontraram-se valores (correlações de Pearson) superiores a 0,20 (Clark \& Watson, 1995), com a maioria dos valores próxima a 0,40 , que atesta a adequação do conjunto de itens para mensurar com consistência os construtos correspondentes. Prova adicional dessas evidências ficou por conta da confiabilidade composta, que tem a vantagem de não ser influenciada pelo número de itens da medida. Neste caso, observaram-se valores acima de 0,60, atendendo recomendação da literatura (Škerlavaj \& Dimovski, 2009). Portanto, parece clara a adequação desta escala em termos de precisão.

\section{Considerações finais}

Tomando em conta o conjunto de resultados previamente apresentados, constatam-se evidências que dão conta da validade de construto (validade fatorial, validade convergente e validade discriminante) $\mathrm{e}$ precisão (consistência interna, homogeneidade e confiabilidade composta) da Escala de Procrastinação Ativa. Portanto, justifica-se seu emprego no contexto brasileiro, quando o propósito for medir a procrastinação ativa das pessoas, conhecendo seus correlatos.

Entretanto, não se encerram as possibilidades de estudos com esta medida. No caso, podem ser pensadas pesquisas futuras que venham a agregar evidências de sua validade e precisão. Por exemplo, caberá avaliar em que medida esta escala é diferente de outras que avaliam tradicionalmente a procrastinação, enfocando em seu aspecto negativo (Ferrari, Johnson \& McCown, 1995). Nesse caso, caberia realizar estudo para apreender se elas apresentam validade discriminante. É possível que a procrastinação ativa tenha reflexo específico quanto à realização, sobretudo no contexto escolar; assim, valerá a pena conhecer em que medida ela explicará (validade preditiva) as pontuações de jovens estudantes. Caberá, igualmente, conhecer se este instrumento apresenta estabilidade temporal (teste-reteste), o que favorecerá seu emprego, por exemplo, quando a situação demandar diferentes medidas ao longo do tempo. Por fim, testar se esta escala apresenta parâmetros similares em outros contextos culturais, quer no Brasil, quer em outros países, parece também um frente de pesquisa promissora, considerando que ainda são escassos os achados sobre esta medida (Choi \& Moran, 2009).

\section{Referências}

Akerlof, G. A. (1991). Procrastination and obedience. The American Economic Review, 81, 1-19.

Bernold, L. E. (2007). Preparedness of engineering freshman to inquiry-based learning. Journal of Professional Issues in Engineering Education and Practice, 133, 99-106.

Biblioteca Virtual em Saúde. (2012). Procrastinação. Disponível em http://www.bireme.br/php/index.php

Boice, R. (1996). Procrastination and blocking: a novel, practical approach. Westport: Preager.

Bui, N. H. (2007). Effect of evaluation threat on procrastination behavior. The Journal of Social Psychology, 147, 197-209.

Psico-USF, Bragança Paulista, v. 19, n. 2, p. 345-354, maio/agosto 2014 
Byrne, B. M. (2010). Structural equation modeling with AMOS: basic concepts, applications, and programming. Nova Iorque: Routledge.

Choi, J. N., \& Moran, S. V. (2009). Why not procrastinate? Development and validation of a new active procrastination scale. The Journal of Social Psychology, 149, 195-211.

Chu, A. H. C., \& Choi, J. N. (2005). Rethinking procrastination: positive effects of "active" procrastination behavior on attitudes and performance. The Journal of Social Psychology, 145, 245-264.

Clark, L. A., \& Watson, D. (1995). Constructing validity: Basic issues in objective scale development. Psychological Assessment, 7, 309-319.

Ellis, A., \& Knaus, W. (1977). Overcoming procrastination. Nova Iorque: Institute for Rational Living.

Enumo, S. R. F., \& Kerbauy, R. R. (1999). Procrastinação: descrição de comportamentos de estudantes e transeuntes de uma capital brasileira. Revista Brasileira de Terapia Comportamental e Cognitiva, 1, 125-133.

Fee, R. L., \& Tangney, J. P. (2000). Procrastination: a means of avoiding shame or guilt? Journal of Social Behavior and Personality, 15, 167-184.

Ferrari, J. R., Johnson, J. L., \& McCown, W. G. (1995). Procrastination and task avoidance: theory, research, and treatment. Nova Iorque: Plenum.

Freedman, J. L., \& Edwards, D. R. (1988). Time pressure, task performance, and enjoyment. Em J. E. McGrath (Ed.), The social psychology of time (pp. 113133). Beverly Hills, CA: Sage Publications.

Gouveia, V. V. (2013). Teoria funcionalista dos valores bumanos: fundamentos, aplicações e perspectivas. São Paulo: Casa do Psicólogo.

Gouveia, V. V., Guerra, V. M., Sousa, D. M. F., Santos, W. S. \& Costa, J. M. (2009). Escala de Desejabilidade Social de Marlowe-Crowne: evidências de sua validade fatorial e consistência interna. Avaliação Psicológica, 8, 87-98.

Gouveia, V. V., Sousa, D. M. F. D., Albuquerque-Souza, A. X. D., Sá-Serafim, R. C. D. N., \& Gonçalves, C. M. T. D. S. (2011). Escala de atitude frente à fofoca: evidências de validade e confiabilidade. Psicologia Ciência e Profissão, 31, 616-627.

Hair, Jr., J. F., Black, W. C., Babin, B. J., Anderson, R. E., \& Tatham, R. L. (2009). Multivariate data analysis
(6 ${ }^{a}$ ed.). Upper Saddle River, NJ: Pearson Prentice Hall.

Hamasaki, E. I. M., \& Kerbauy, R. R. (2001). Será o comportamento de procrastinar um problema de saúde? Revista Brasileira de Terapia Congitiva Comportamental, 3, 35-40.

Kerbauy, R. (1997). Procrastinação: Adiamento de tarefas. Em R. A. Banaco (Org.), Sobre o comportamento e cognição: aspectos teóricos, metodológicos e de formação em análise do comportamento clínico e terapia cognitiva (pp. 393-398). Santo Andre, SP: ESETec Editores Associados.

Knaus, W. J. (2000). Procrastination, blame, and change. Journal of Social Behavior and Personality, 15, 153-166.

Milgram, N. A., Sroloff, B., \& Rosenbaum, M. (1988). The procrastination of everyday life. Journal of $R e-$ search in Personality, 22, 197-212.

Nunnally, J. C. (1991). Teoría psicométrica. México, DF: Trilhas.

Pasquali, L. (2003). Psicometria: teoria dos testes na psicologia e na educação. Petrópolis, RJ: Vozes.

Potts, T. J. (1987). Predicting procrastination on academic tasks with self-report personality measures. Dissertation Abstracts International, 48, 1543.

PsychINFO (2013). Procrastinação. Disponível em http:// www.psychinfo.com

Silva, W. M., \& Yu, A. S. O. (2009). Análise empírica do senso de controle: Buscando entender o excesso de confiança. Revista de Administração Contemporânea, 13, 247-271.

Škerlavaj, M., \& Dimovski, V. (2009). Organizational learning and performance in two national cultures: A multi-group structural equation modeling approach. Em W. R. King (Ed.), Knowledge management and organizational learning (pp. 321-366). Nova Iorque: Springer.

Solomon, L.J., \& Rothblum, E.B. (1984). Academic procrastination: frequency and cognitive behavioural correlates. Journal of Counseling Psychology, 31, 503-509.

Steel, P. (2007). The nature of procrastination: a meta-analytic and theoretical review of quintessential self-regulatory failure. Psychological Bulletin, 133, 65-94. 
Strongman, K. T., \& Burt, C. D. B. (2000). Taking

Recebido em: 23/05/2013

breaks from work: an exploratory inquiry. Journal

Reformulado em: 25/11/2013

of Psychology, 134, 229-242.

Aprovado em: 17/03/2014

Tabachnick, B. G., \& Fidell, L. S. (2013). Using multivariate statistics ( $6^{\mathrm{a}}$ ed.). Nova Iorque: Allyn \& Bacon.

Nota dos autores:

O presente artigo contou com apoio do CNPq por meio de bolsa de Produtividade em Pesquisa concedida ao primeiro autor. Os autores agradecem a essa instituição.

Sobre os autores:

Valdiney V. Gouveia é professor titular de Psicologia Social na Universidade Federal da Paraíba (http://vvgouveia. net), e pesquisador Nível $1 \mathrm{~A}$ do $\mathrm{CNPq}$, atuando como consultor de agências nacionais e internacionais de fomento à pesquisa.

Viviany da Silva Pessoa é licenciada em Psicologia, mestre em Psicologia (UFRN) e doutora em Psicologia Social (UFPB). Atualmente é professora adjunta do Curso de Psicopedagogia da Universidade Federal da Paraíba, atuando nas áreas de avaliação, relação pessoa-ambiente, linguagem e cognição.

Marcio de Lima Coutinho é doutor em Psicologia Social (UFPB). Atualmente é professor da Pós-Graduação no Centro Universitário de João Pessoa, atuando como professor de Métodos de Pesquisa e Estatística e pesquisador em Psicologia Social com ênfase na abordagem quantitativa.

Iany C. da S. Barros é graduada em Psicologia (FAFIRE), mestre em Educação e doutoranda em Psicologia Social (UFPB). Atualmente é Professora Titular do Centro Universitário de João Pessoa, exercendo a função de Pró-reitora de Pós-graduação, pesquisa e extensão no Centro Univesitario de Joao Pessoa - UNIPE.

Aline Arruda da Fonseca é psicóloga, mestre em Psicologia e doutoranda em Psicologia Social (UFPB). Atualmente é professora da Faculdade Maurício de Nassau, atuando nas áreas de Desenvolvimento Humano, Avaliação Psicológica e Psicopatologia.

Contato com os autores:

Valdiney Veloso Gouveia

Universidade Federal da Paraíba, CCHLA

- Departamento de Psicologia -

CEP: 58.051-900, João Pessoa - PB.

E-mail:vvgouveia@gmail.com. 